\title{
Quantifying the burden of caregiving in Duchenne muscular dystrophy
}

\author{
Erik Landfeldt ${ }^{1,2} \cdot$ Peter Lindgren $^{3} \cdot$ Christopher F. Bell $^{4} \cdot$ Michela Guglieri $^{5}$ • \\ Volker Straub $^{5} \cdot$ Hanns Lochmüller ${ }^{5}$ Katharine Bushby ${ }^{5}$
}

Received: 12 November 2015/Revised: 14 January 2016/Accepted: 16 January 2016/Published online: 10 March 2016 (C) The Author(s) 2016. This article is published with open access at Springerlink.com

\begin{abstract}
Duchenne muscular dystrophy (DMD) is a rare pediatric neuromuscular disease associated with progressive muscle degeneration and extensive care needs. Our objective was to estimate the caregiver burden associated with DMD. We made cross-sectional assessments of caregiver health-related quality of life (HRQL) and burden using the EuroQol EQ-5D, a Visual Analogue Scale (VAS), the SF-12 Health Survey, and the Zarit Caregiver Burden Interview (ZBI) administered online. Results were stratified by disease stage (early/late ambulatory/non-ambulatory) and caregivers' rating of patients' health and mental status. In total, caregivers to 770 patients participated. Mean EQ-5D utility ranged between $0.85(95 \% \mathrm{CI}$
\end{abstract}

H. Lochmüller and K. Bushby contributed equally to the manuscript.

Electronic supplementary material The online version of this article (doi:10.1007/s00415-016-8080-9) contains supplementary material, which is available to authorized users.

Erik Landfeldt

erik.landfeldt@ki.se

1 Institute of Environmental Medicine, Karolinska Institutet, Nobels väg 13, SE-17177 Stockholm, Sweden

2 OptumInsight, Klarabergsviadukten 90, SE-11164 Stockholm, Sweden

3 Medical Management Centre, Department of Learning, Informatics, Management and Ethics, Karolinska Institutet, Tomtebodavägen 18A, SE-17177 Stockholm, Sweden

4 GlaxoSmithKline, 5 Moore Drive, PO Box 13398, Research Triangle Park, Durham, NC 27709-3398, USA

5 Newcastle University John Walton Muscular Dystrophy Research Centre and MRC Centre for Neuromuscular Diseases, Institute of Genetic Medicine, International Centre for Life, Central Parkway, Newcastle upon Tyne NE1 3BZ, UK
$0.82-0.88)$ and $0.77(0.74-0.80)$ across ambulatory classes and $0.88(0.85-0.90)$ and $0.57(0.39-0.74)$ across caregivers' rating of patients' health and mental status. Mean VAS score was 0.74 (0.73-0.75), mean SF-12 Mental Health Component Summary score 44 (43-45), and mean ZBI score 29 (28-30). Anxiety and depression, recorded in up to $70 \%$ of caregivers depending on patients' health and mental status, was significantly associated with annual household cost burden $(>\$ 5000$ vs. $<\$ 1000$, odds ratio $1.76,95 \%$ CI 1.18-2.63) and hours of leisure time devoted to informal care per week $(25-50$ vs. $<25 \mathrm{~h} 2.01$, $1.37-2.94$; $>50$ vs. $<25$ h $3.35,2.32-4.83)(p<0.007)$. We show that caring for a person with DMD can be associated with a substantial burden and impaired HRQL. Our findings suggest that caregivers to patients with DMD should be screened for depression and emphasize the need for a holistic approach to family mental health in the context of chronic childhood disease.

Keywords Caregiver burden - Quality of life - Utilities · Informal care $\cdot$ Neuromuscular

\section{Introduction}

Throughout the Western world, including the US, Australia, Japan, and the European Union, long-term care of disabled or chronically ill patients is predominately provided at home by untrained, unpaid family members [1-4]. For many caregivers, assisting their spouse, parent, offspring, or other relative in their day-to-day life may be a positive experience, but can at times also be tremendously challenging. Numerous studies have shown that the provision of informal care is associated with serious adverse health effects for the caregiver, including anxiety and 
depression, impaired immune system function, and coronary heart disease, as well as social isolation, financial deprivation, and even premature death [1, 5-7]. Existing research, however, focus on the burden on caregivers to elderly patients, predominantly with dementia, with less attention devoted to genetic pediatric conditions, including Duchenne muscular dystrophy (DMD).

DMD is a rare, $\mathrm{X}$-linked, neuromuscular disease characterized by progressive muscle weakening, diminishing functional ability, and serious multisystem complications, with a mean life expectancy of 25 years [8-10]. As a result of the devastating disease progression, patients inevitably transition towards a state of total dependency, requiring wheelchairs for mobility from their early teens and ventilation support for survival in more advanced stages of the disease. Many patients with DMD also suffer from mental health comorbidities, such as autism spectrum disorder (ASD) and obsessive-compulsive disorder (OCD). The complexity of the disease necessitates multidisciplinary management including regular visits to neuromuscular, cardiac, and respiratory specialists, physiotherapists, and other healthcare practitioners $[8,9]$.

We have previously reported that caring for a child with DMD is both time-consuming and financially burdensome [11]. Many DMD caregivers terminate their employment or reduce their working hours to find the time needed to care for their sons, and those who do continue to work have markedly impaired productivity with high levels of absenteeism. In addition to forgone income, depending on national policies, affected households also carry substantial costs associated with, e.g., insurance premiums and copayments for healthcare.

The caregiver burden has been studied in DMD only in small samples from a single clinic or country, in combination with other muscle dystrophies, and/or without stratifying results by disease stage or patient health status [12-22]. The objective of this multinational study was to complement our previous data on the objective caregiver burden in DMD with estimates of the subjective burden: that is, the impact on health-related quality of life (HRQL). A specific aim was to investigate mental distress among caregivers.

\section{Methods}

\section{Participants and procedures}

Caregivers to patients with DMD were recruited as part of a multinational, cross-sectional, observational study for which details and results have been previously reported $[11,23]$. In summary, patients with DMD from Germany, Italy, the UK, and the US were recruited through national
DMD registries which form part of the global TREATNMD network [24]. To be eligible, patients were required to fulfill the following criteria: (1) male, (2) DMD diagnosis, and (3) age $\geq 5$ years. Caregivers to eligible patients were invited to complete a questionnaire online. All participants provided informed consent and study approval was granted from Ludwig-Maximilians-Universität München (Germany), Comitato Etico IRCCS E. Medea, Associazione La Nostra Famiglia (Italy), North East Research Ethics Service, NHS (UK), the Western Institutional Review Board (US), and the TREAT-NMD Global Databases Oversight Committee.

\section{Outcome measures}

We assessed caregiver HRQL using the EuroQol EQ-5D-3L (EQ-5D) [25], a Visual Analogue Scale (VAS), and the SF-12 Health Survey (SF-12) [26]. The EQ-5D is a generic HRQL instrument encompassing five dimensions (mobility, self-care, usual activities, pain/discomfort, and anxiety/depression), each described in three levels. EQ-5D outcomes are linked to preference values, known as utilities ranging from $0=$ dead to $1=$ perfect health, derived from the general public. The VAS was presented as a continuous response scale, ranging from $0=$ "worst imaginable health" to $1=$ "best imaginable health", measuring self-perceived HRQL.

The SF-12 is a generic HRQL instrument comprising 12 questions, each described in three to five levels. SF-12 outcomes include two composite scores, the Physical Component Summary Score (PCS) and the Mental Health Component Summary Score (MCS), as well as eight separate scores. The instrument uses norm-based scoring (mean $=50, \mathrm{SD}=10)$ and values $<43$ or $>56$ are considered significantly different from the general population [26].

In addition to the HRQL instruments, we assessed the caregiver burden using the Zarit Caregiver Burden Interview (ZBI) [27]. The ZBI contains 22 questions, each described in five levels (global score range from $0=$ low burden to $88=$ high burden).

To investigate the possible association between patient and caregiver HRQL, we also asked caregivers to rate their sons' current health (categories ranging from "excellent" to "poor") and mental status (categories ranging from "happy and interested in life" to "very unhappy").

\section{Statistical analysis}

We assessed mean EQ-5D utility using the recommended and most robust valuation set derived through the timetrade-off method [25] (results for other EQ-5D valuation sets presented as supplemental material online), mean VAS scores, mean global caregiver ZBI scores, and mean SF-12 PCS and MCS scores. We assessed and reported EQ-5D 
results for anxiety and depression separately as we hypothesized that this domain would be most influenced by the caregiver role.

We related our results to the progression of DMD by classifying patients into four groups defined first in terms of current ambulatory status and second in terms of age: (1) early ambulatory (approx. age 5-7 years), (2) late ambulatory (approx. age 8-11 years), (3) early non-ambulatory (approx. age 12-15 years), and (4) late non-ambulatory (approx. 16 years of age, or older) [8, 9].

We compared our estimates with EQ-5D and VAS general population reference data [28, 29] using Welch's $t$ tests and Welch's analysis-of-variance models. We fitted five logistic regression models to test for differences in anxiety and depression across patients' ambulatory status (model I), caregivers' ratings (model II and III), and two objective measures of the caregiver burden (annual household cost burden and hours of leisure time devoted to informal care [11]) (model IV and V). Goodness-of-fit was assessed using Hosmer and Lemeshow's test. All analyses were conducted in Stata 14.

\section{Results}

Demographic statistics of the participating caregivers ( $n=770)$ are presented in Table 1. Patients had a mean age of 14 years (median 12 years; interquartile range 9-17 years), $47 \%$ (359 of 770) were wheelchair dependent, $16 \%$ (126 of 770) required ventilation support, and $63 \%$ (486 of 770) were currently taking glucocorticoids. Additional details of the study sample have been previously published $[11,23]$.

\section{Prevalence of anxiety and depression}

Half of all caregivers (383 of 770) reported being moderately or extremely anxious or depressed, significantly higher than general population reference data for individuals aged 40-49 years across all investigated strata ( $p<0.001$ for all comparison) (Fig. 1). Adjusted logistic regression results showed that anxiety and depression was strongly associated with the caregivers' rating of patients' health and mental status, as well as measures of objective burden (i.e., annual household cost burden and hours of leisure time devoted to informal care), but not ambulatory class (Table 2). The prevalence of anxiety and depression was comparable across countries $(p=0.139)$.

\section{Caregiver health-related quality of life}

Mean EQ-5D utilities, ranging from $0=$ dead to $1=$ perfect health representing general public preferences
Table 1 Demographic statistics of the DMD caregivers $(n=770)$

\begin{tabular}{|c|c|}
\hline & $n($ proportion $\%)$ \\
\hline \multicolumn{2}{|l|}{ Country of residence } \\
\hline Germany & $173(22)$ \\
\hline Italy & $122(16)$ \\
\hline The UK & $191(25)$ \\
\hline The US & $284(37)$ \\
\hline Sex, female & $609(79)$ \\
\hline Age, mean (SD) (years) & $44(8)$ \\
\hline University degree & $324(42)$ \\
\hline \multicolumn{2}{|l|}{ Marital status } \\
\hline Married/partner & $656(85)$ \\
\hline Separated/divorced & $75(10)$ \\
\hline Single & $30(4)$ \\
\hline Widowed & $9(1)$ \\
\hline \multicolumn{2}{|l|}{ Relationship to the patient } \\
\hline Parent & $753(98)$ \\
\hline Other relative, friend, or partner & $17(2)$ \\
\hline \multicolumn{2}{|l|}{ Current situation } \\
\hline Employed & $469(61)$ \\
\hline Unemployed & $257(33)$ \\
\hline Retired & $26(3)$ \\
\hline Student & $12(2)$ \\
\hline Sick leave ( $>3$ months) & $6(1)$ \\
\hline \multicolumn{2}{|l|}{ Household income class ${ }^{\mathrm{a}}$} \\
\hline Poor & $72(9)$ \\
\hline Middle class & $615(80)$ \\
\hline Rich & $83(11)$ \\
\hline \multicolumn{2}{|l|}{ Annual household cost burden ${ }^{\mathrm{b}}$} \\
\hline$<\$ 1000$ & $380(49)$ \\
\hline$\$ 1000-\$ 5000$ & $170(22)$ \\
\hline$>\$ 5000$ & $220(29)$ \\
\hline \multicolumn{2}{|c|}{ Hours of leisure time devoted to informal care (per week) } \\
\hline$<25$ & $294(38)$ \\
\hline $25-50$ & $203(26)$ \\
\hline$>50$ & $273(35)$ \\
\hline Additional household member with DMD & $55(7)$ \\
\hline
\end{tabular}

Because of rounding, percentages might not add up to $100 \%$ exactly

a Poor income class: $<60 \%$ of national median equalized household disposable income; rich income class: $>200 \%$ of national median equalized household disposable income

b Include non-reimbursed payments for insurance premiums, copayments for medical and community services and medications, and out-of-pocket payments for investments (e.g., non-reimbursed payments for medical and non-medical aids and devices and investments to and reconstructions of the home)

of HRQL, are presented in Fig. 2. The sex- and age-matched loss in caregiver utility in relation to the general population was estimated at between 0.09 (95\% CI $0.07-0.11)$ and $0.14(0.11-0.17)$ across ambulatory classes, between $0.06(0.04-0.07)$ and $0.18(0.13-0.23)$ across 


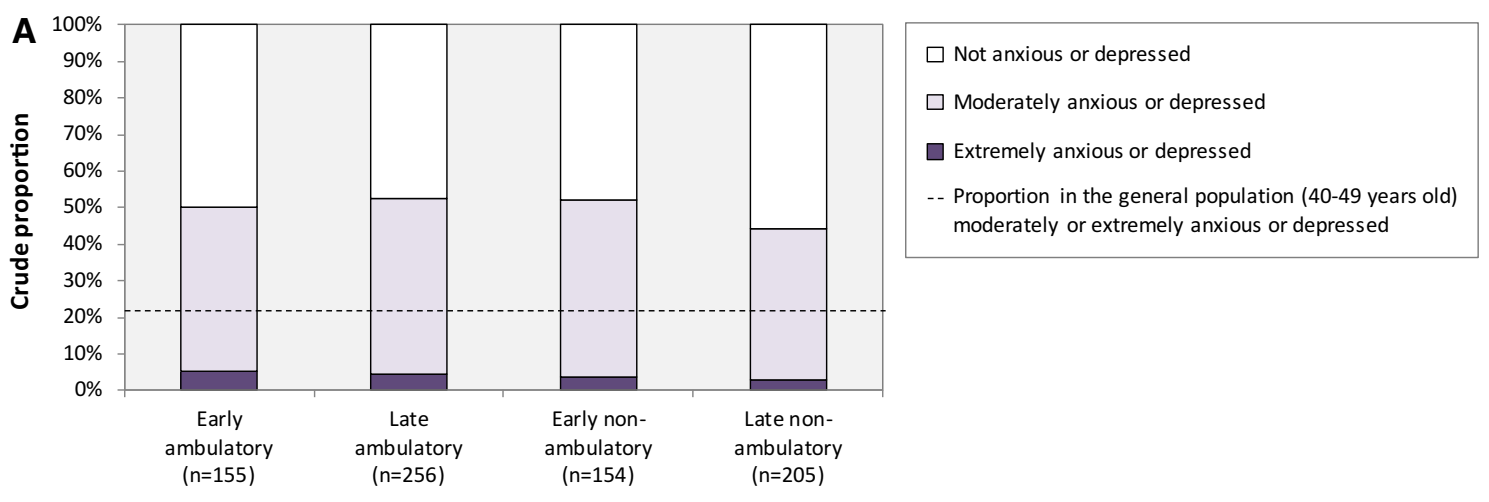

Ambulatory status of DMD patient

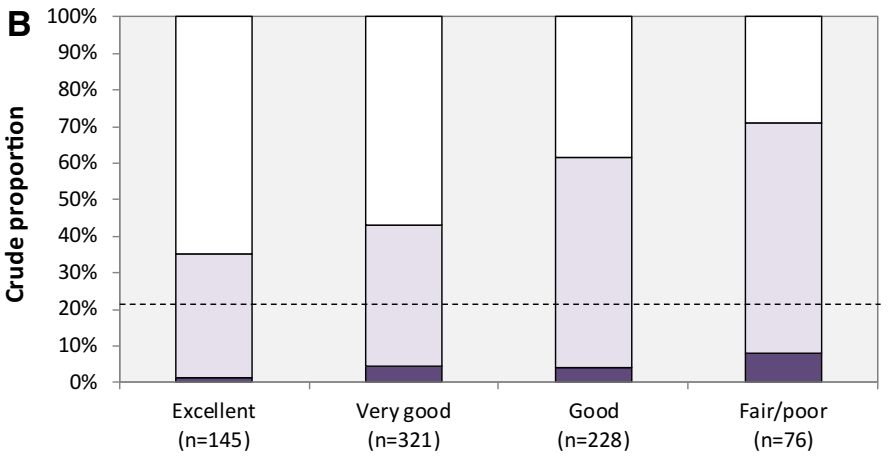

Caregivers' rating of their sons' current health

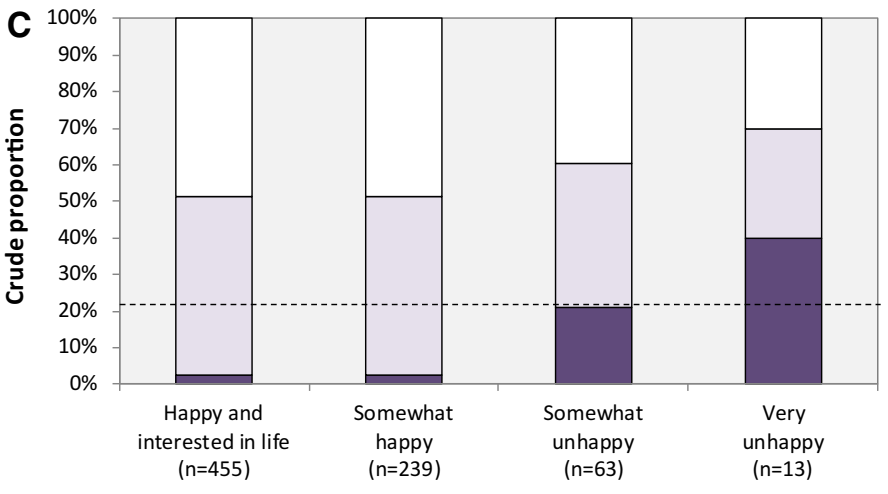

Caregivers' rating of their sons' current mental status

Fig. 1 Prevalence of anxiety and depression in DMD caregivers

caregivers' rating of their sons' current health, and between 0.09 (0.07-0.10) and $0.30(0.13-0.46)$ across caregivers' rating of their sons' current mental status. Compared with general population reference data for individuals aged 40-49 years, a significantly larger proportion of DMD caregivers reported having pain or discomfort (44 vs. $33 \%$, $p<0.001)$ and problems performing usual activities (18 vs. $16 \%, p=0.006)$. Additional EQ-5D utility results available as supplemental material online.

Mean VAS scores, representing the caregivers' subjective rating of their own HRQL ranging from $0=$ "worst imaginable health" to $1=$ "best imaginable health", was lower than the estimated EQ-5D utilities in all strata, except for caregivers to patients rated to be very unhappy (Fig. 2). Neither mean utilities nor VAS scores were significantly different across countries.

Mean SF-12 MCS score, ranging from 0 to 100 where higher score reflects higher HRQL, was estimated at 44 (95 $\%$ CI 43-45), ranging between 44 (42-45) and 46 (45-48) across ambulatory classes, 48 (47-50) and 37 (35-40) across the caregivers' rating of their sons' current heath, and 46 (45-47) and 33 (26-40) across the caregivers' rating of their sons' current mental status. Mean PCS scores were within the normal range in all strata. 
Table 2 Predictors of anxiety and depression in DMD caregivers

\begin{tabular}{|c|c|c|c|}
\hline & $n$ & Odds ratio $(95 \% \mathrm{CI})^{\mathrm{a}}$ & $p$ value \\
\hline \multicolumn{4}{|c|}{ Model I: patients' ambulatory status } \\
\hline Early ambulatory & 155 & 1 & \\
\hline Late ambulatory & 256 & $1.08(0.70-1.65)$ & 0.742 \\
\hline Early non-ambulatory & 154 & $1.04(0.64-1.70)$ & 0.873 \\
\hline Late non-ambulatory & 205 & $0.93(0.53-1.64)$ & 0.807 \\
\hline \multicolumn{4}{|c|}{ Model II: caregiver perceptions' of patients' health } \\
\hline Excellent & 145 & 1 & \\
\hline Very good & 321 & $1.53(1.00-2.33)$ & 0.049 \\
\hline Good & 228 & $3.85(2.40-6.20)$ & $<0.001$ \\
\hline Fair/poor & 76 & $5.87(3.05-11.29)$ & $<0.001$ \\
\hline \multicolumn{4}{|c|}{ Model III: caregivers' perception of patients' mental status } \\
\hline Happy and interested in life & 455 & 1 & \\
\hline Somewhat happy & 239 & $1.85(1.32-2.58)$ & $<0.001$ \\
\hline Somewhat unhappy & 63 & $4.67(2.44-8.92)$ & $<0.001$ \\
\hline Very unhappy & 13 & $7.22(1.79-29.09)$ & 0.005 \\
\hline \multicolumn{4}{|c|}{ Model IV: annual household cost burden } \\
\hline$<\$ 1000$ & 380 & 1 & \\
\hline$\$ 1000-\$ 5000$ & 170 & $1.43(0.95-2.16)$ & 0.090 \\
\hline$>\$ 5000$ & 220 & $1.76(1.18-2.63)$ & 0.006 \\
\hline \multicolumn{4}{|c|}{ Model V: hours of leisure time devoted to informal care (per week) } \\
\hline$<25$ & 294 & 1 & \\
\hline $25-50$ & 203 & $2.01(1.37-2.94)$ & $<0.001$ \\
\hline$>50$ & 273 & $3.35(2.32-4.83)$ & $<0.001$ \\
\hline
\end{tabular}

\section{Subjective caregiver burden}

The mean global ZBI score, ranging from $0=$ low burden to $88=$ high burden, was estimated at 29 (95\% CI 28-30), ranging between $25(23-27)$ and 32 (30-34) across ambulatory classes, $23(21-25)$ and 38 (34-41) across the caregivers' rating of sons' current health, and 26 (24-27) and 41 (34-48) across the caregivers' rating of sons' current mental status. Results from the ZBI, sorted by score (i.e., extent of caregiver burden) for each question, are presented in Fig. 3. Additional ZBI results available as supplemental material online.

\section{Discussion}

Caregiver burden has received considerable attention in the gerontology literature during recent decades, but relatively few studies have investigated the impact on HRQL in caregivers to children with chronic diseases. Compared with adult caregivers of patients with diseases associated with advanced age, such as dementia or Parkinson's, parents raising a child with a chronic illness may face even greater challenges as they normally live together and have no choice but to fully take on the caregiver role [1]. In addition, as a result of improved survival in many childhood diseases, including DMD, the duration of informal caregiving has increased considerably, in some indications from years to several decades, with increased morbidity and care needs in later stages of the patient's life [30, 31]. For incurable, terminal, progressive conditions, awareness of the devastating disease trajectory would also be expected to have serious adverse effects on caregiver mental well-being, and for genetic diseases, there may also be aspects of guilt.

The objective of this study was to investigate the subjective caregiver burden associated with DMD. Overall, half of all DMD caregivers in our sample indicated being moderately or extremely anxious or depressed, and we also found the mental health summary score from the SF-12 to be significantly lower than general population reference data. These result confirms previous findings of elevated 

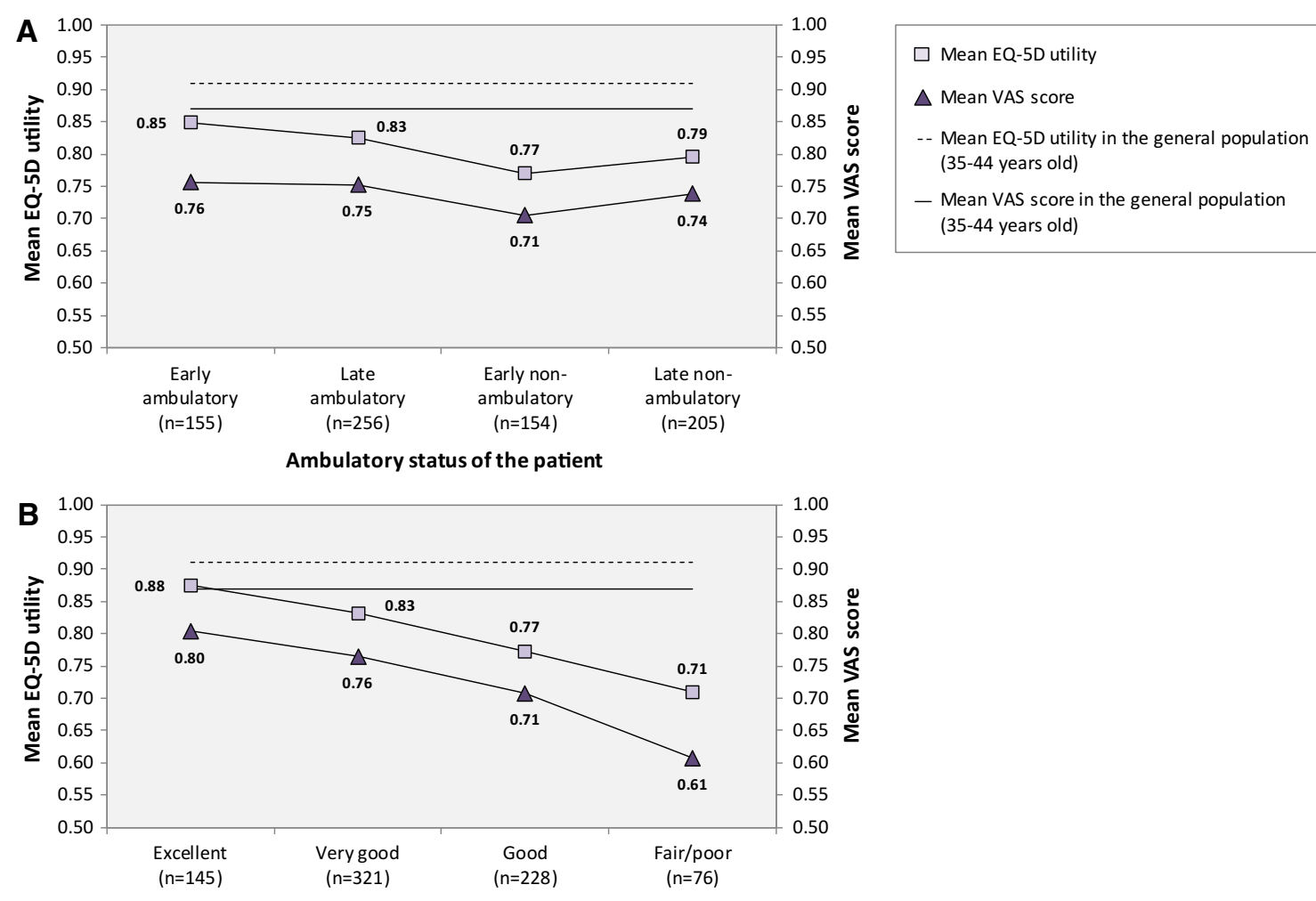

Caregivers' rating of their sons' current health

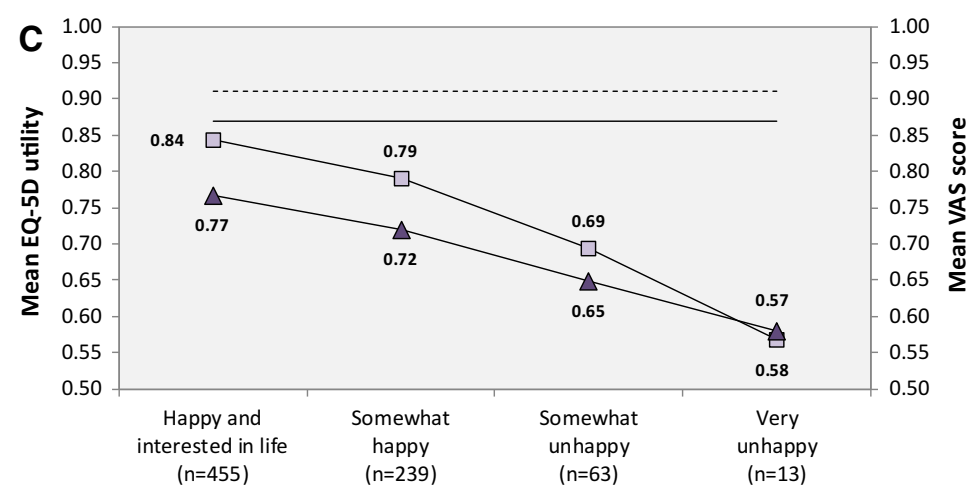

Caregivers' rating of their sons' current mental status

Fig. 2 Caregiver health-related quality of life

risks of depression and distress reported in caregivers to children with DMD and Becker muscular dystrophy [1517], type 1 diabetes [32], and autism [33], as well as parenting stress in pediatric chronic illnesses [34]. We found the prevalence of anxiety and depression to be comparable over the course of disease progression but strongly associated with the health and mental state of the patient as perceived by the caregiver. Specifically, caregivers to patients in fair/poor compared to excellent health had a sixfold risk increase of anxiety and depression. Comparing patients perceived as very unhappy and happy, we noted a sevenfold risk increase. These data suggest that it may be relevant to screen for anxiety and depression in caregivers to patients with DMD, and that patients' health and mental status may be helpful predictors of caregiver distress.

To our knowledge, only one study has previously estimated HRQL in DMD caregivers using the EQ-5D, and in contrast to our findings, this study found the prevalence of anxiety and depression comparable to the general population [20]. Possible reasons for this discrepancy include differences in sample size and patient demographics, as the previous study was based on parents to 57 adult patients with DMD (mean age 27 years).

We found overall caregiver HRQL, as measured by EQ$5 \mathrm{D}$ utilities representing general population preferences, to be markedly impaired and closely associated with patient 


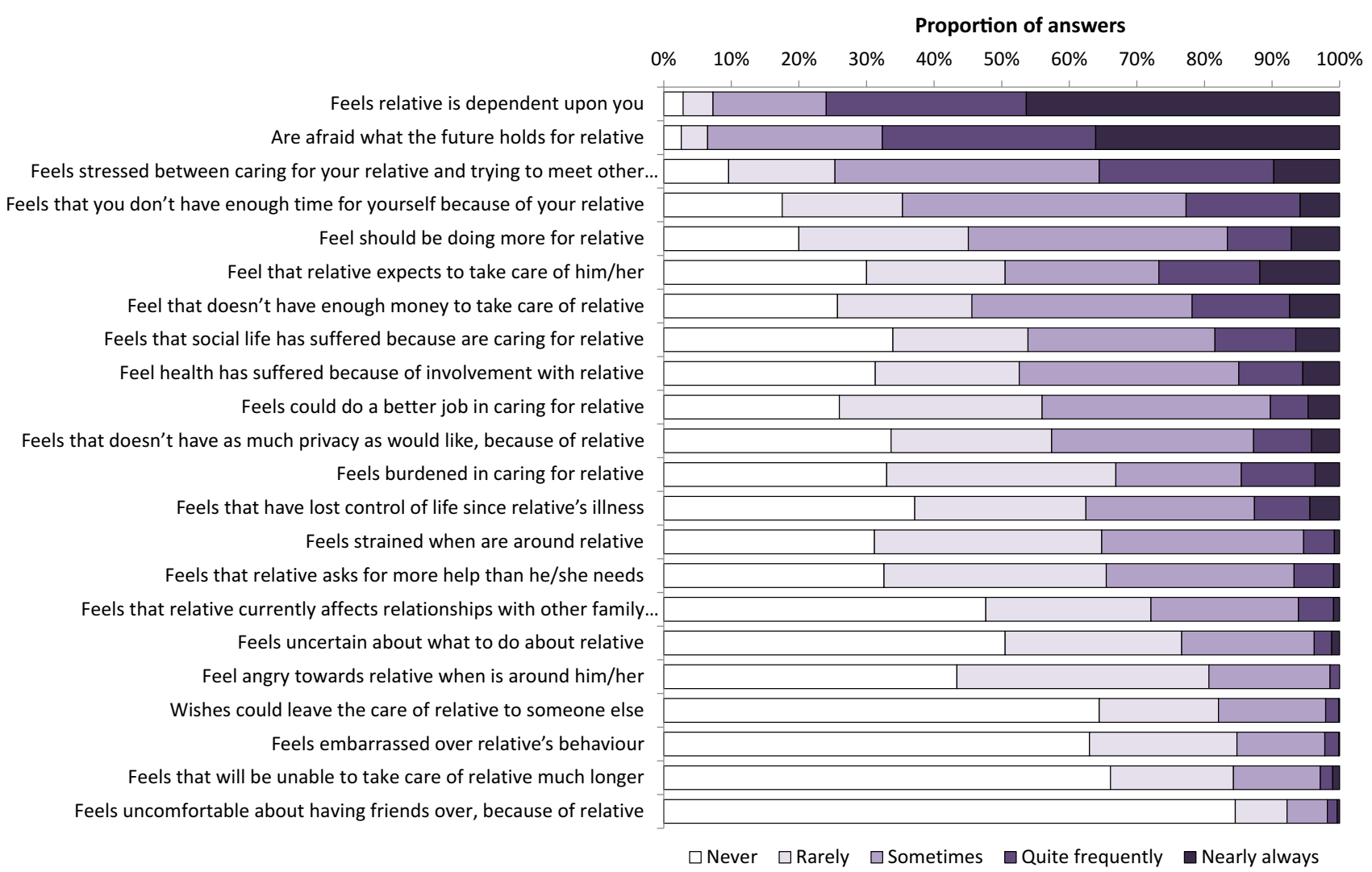

Fig. 3 Distribution of replies from the Zarit Caregiver Burden Interview

health and mental status, but not ambulatory class. Specifically, the mean caregiver utility was recorded at 0.81 , significantly lower than the only previous report in DMD $(0.87, p<0.001)$ [20], and we estimated the mean age- and sex-matched disutility at between 0.06 and 0.30 across the investigated strata (mean in the pooled sample was 0.11). This implies that the loss in HRQL among DMD caregivers was similar to or greater than published disutility estimates for patients suffering from very serious and sometimes rapidly fatal diseases, including lung cancer and schizophrenia (0.11), systemic lupus erythematosus (0.08), and epilepsy (0.07) [35]. Given how insensitive the EQ-5D appears to be to capture impairment in HRQL in many conditions (e.g., breast and prostate cancer, asthma, and myocardial infarction, all with disutilities below the minimally important difference threshold of 0.074 ) $[35,36]$, the considerable loss in utility observed in our study is both surprising and noteworthy, and captures the exquisite stresses associated with caring for a child with a chronically disabling and progressive condition, with an invariably fatal outcome. The small change in utility and VAS scores across ambulatory classes indicates that caregivers may find ways to learn to cope with the disease and the increasing levels of disability and morbidity associated with the progression of DMD, and adjust their perception of their own HRQL over time. As shown in Fig. 2, the mean utility and VAS scores were in fact slightly higher among caregivers to late compared to early non-ambulatory patients (although the differences were not statistically different, $p=0.254$ and 0.113 , respectively), possibly related to additional assistance from, e.g., nurses and other healthcare practitioners when patients become more disabled. Comparing results from the SF-12 and the EQ-5D, it is also worth noting that the former indicated normal physical health, whereas EQ-5D domains relating to pain and discomfort and usual activities were found to be significantly impaired in relation to the general population. These inconsistent results may be explained by differences in the design and scoring of the two instruments, but warrants further research, in particular considering the recognized notion that without mental health there can be no true physical health [37].

Kenneson et al. show that employment outside of the home is a predictor of stress in caregivers to boys with DMD and Becker muscular dystrophy [16]. In line with this finding, across countries, we have previously reported that between 27 and $49 \%$ of caregivers in our sample reduced their working hours or stopped working completely due to their son's DMD, and we have also estimated the mean number of hours of leisure time devoted to informal caregiving per week at between 33 and 44 [11]. Still, in the present study, depending on their rating of 
patient well-being, between 12 and $40 \%$ of caregivers replied that they frequently or always felt that they should be doing more for their son. We also found that between 57 and $86 \%$ of caregivers frequently or always felt worried about the future of their child, 26 and $69 \%$ were stressed between the demands of caring for the relative and trying to meet other responsibilities for family or work, and 17 and $62 \%$ that they did not have enough money to take care of their son. Together with our findings that a high annual household cost burden and $>25$ h of leisure time devoted to informal care per week is associated with anxiety and depression, these data emphasize that lack of resources (i.e., time and money) is an important source of distress in DMD caregivers.

We estimated the mean global ZBI score at 29, ranging from 23 to 41 across investigated strata, which may be compared with estimates from studies in other diseases, for example neuromuscular diseases in general (23) [13], irritable bowel syndrome (22) [38], Alzheimer's disease (29) [39], obsessive-compulsive disorder (29) [40], and Parkinson's disease (24) [41]. Although these findings indicate that caring for a patient with DMD is burdensome, it is difficult to further interpret the results as there is no link between ZBI scores and subjective caregiver burden or well-being. For example, to what extent are caregivers burdened by the feeling that their sons are dependent on them, or that they feel embarrassed over their sons' behavior, or by not having as much privacy as they would like? This would be expected to vary across caregivers, by age, sex, cultural setting, and a range of other factors. In other words, the ZBI scoring algorithm does not take into account that the questions and response categories included in the instrument may have a different impact on the selfperceived burden of each caregiver.

Despite the negative impact on well-being, there is also evidence that being a caregiver to an individual with DMD can be a positive, rewarding experience. For example, Pangalila et al. found that $95 \%$ of DMD caregivers in their sample regarded caring as enjoyable, and Magliano et al. found that $88 \%$ of caregivers to patients with muscle dystrophies had got something positive out of the situation $[18,20]$. We did not specifically measure positive aspects of caregiving in DMD, but we found some support for these previous results in the ZBI, where $82 \%$ of caregivers never/rarely wished that they could leave the care of their child to someone else, indicative of the devotion with which these caregivers take on their responsibilities, despite the levels of stress and demand.

Although the observational nature of our data prevents us from drawing conclusions of causality, our results have several implications for health policy. First, given the association between number of hours spent providing informal care and caregiver mental health, respite care and similar initiatives is urgently needed to help reduce the family burden and improve caregiver well-being. Second, our results suggest that many families caring for a person with DMD require increased financial support to help shoulder the considerable cost burden associated with the disease, of particular importance given the association between annual household costs and caregiver anxiety and depression demonstrated in this study. It should be noted however that some family caregivers may increase their spending because they are feeling depressed or anxious (to help improve the situation of their child), not vice versa. Third, psychosocial support for caregivers and families must be improved, especially given the poor coverage of these types of services among patients as described in our previous work [23].

Primary strengths of our study include a multinational sample, comprising patients with DMD only (as compared to mixed cohorts of neuromuscular disorders), of sufficient power to enable meaningful stratification across both disease stage and patients' perceived health and mental status. We found patient clinical data to be characteristic for the different ambulatory classes and the distribution of age similar for responders and non-responders (not reported), indicating that any discrepancy between our patient sample and the general DMD population would be limited. We chose to recruit patients via the TREATNMD national DMD registries, which accept registration on a voluntary basis from patients and families with a mutationconfirmed diagnosis of DMD. Although participation in the registries is family initiated, and therefore more likely to be sought by motivated families, the registry-based approach allowed an unbiased sample to be obtained with respect to attendance at any one clinic or restriction to any one domain with potentially different care practices. Our questionnaire was returned with a mean response rate of $42 \%$ which is comparable to other surveys sent out via this kind of route: indeed it is also worth pointing out that the response rate among those who actually received a study invitation would be expected to be notably higher as a result of, e.g., lost invitations due to recent changes to email addresses and spam filters. This also means that our burden estimates should be viewed as conservative, as very distressed caregivers may not have had time nor energy to participate. Despite some limitations in the methodology therefore we believe that this strategy has given us the best possible chance of a generalizable view of burden of care in DMD and an unprecedented glimpse into a large sample size in a rare disease.

\section{Conclusions}

We show that caring for a person with DMD can be associated with a substantial burden and markedly impaired HRQL. Our data underscore the need for healthcare practitioners involved in the medical 
management of DMD to also pay attention to caregiver mental health, in particular when the health and mental status of the patient is perceived as poor. Our findings emphasize the need for a holistic approach to family mental health in the context of chronic childhood disease.

Acknowledgments This study was sponsored by GlaxoSmithKline (study number HO-11-794). The authors gratefully acknowledge Maggie C. Walter, MD, MA, Simone Thiele, and Olivia Schreiber, MD (Friedrich-Baur-Institute, Dept. of Neurology, Ludwig-Maximilians-University, Munich, Germany), Filippo Buccella, Pharm. D, Fernanda G. De Angelis, PhD, Francesca Ceradini, PhD, and Andrea Negroni, ICT Solution Architect (Registro Pazienti DMD/BMD Italia, Parent Project Onlus), Angela Stringer (DMD Registry, Action Duchenne), Emma Heslop, MSc (TREAT-NMD), and Holly L. Peay, MS, CGC, and Ann S. Martin, MS, CGC (DuchenneConnect) for their contribution to the set-up of the study and patient recruitment. We acknowledge support for TREAT-NMD from the European Commission through FP6 (Contract Number 036825) and the Second Public Health Programme (Contract Number 2012 3307), as well as support from the AFM (Contract Number 16104). The German DMD registry acknowledges additional support from the German Duchenne Parent Project "aktion benni \& co" (http://www.benniundco.eu), and the Deutsche Gesellschaft für Muskelkranke (DGM) e.V. (http:// www.dgm.org). The UK DMD Registry acknowledges support and funding from Action Duchenne, a patient led charity. The DuchenneConnect Registry acknowledges support and funding from Parent Project Muscular Dystrophy (PPMD), the largest non-profit organization in the United States focused on finding a cure for Duchenne. We also acknowledge funding from the Medical Research Council as part of the MRC Centre for Neuromuscular Diseases (Reference G1002274, Grant ID 98482).

\section{Compliance with ethical standards}

Conflicts of interest Mr. Landfeldt has acted as a consultant to GlaxoSmithKline through his employment at OptumInsight but was not compensated for his contributions as an author on this manuscript. Dr. Lindgren has acted as a consultant to GlaxoSmithKline. Mr. Bell is an employee and shareholder of GlaxoSmithKline. Dr. Guglieri, Dr. Straub, and Dr. Bushby have been contracted by GSK to serve as investigators in clinical trials with no personal reimbursement. Dr. Lochmüller reports no conflict of interest.

Open Access This article is distributed under the terms of the Creative Commons Attribution 4.0 International License (http://crea tivecommons.org/licenses/by/4.0/), which permits unrestricted use, distribution, and reproduction in any medium, provided you give appropriate credit to the original author(s) and the source, provide a link to the Creative Commons license, and indicate if changes were made.

\section{References}

1. Adelman RD, Tmanova LL, Delgado D, Dion S, Lachs MS (2014) Caregiver burden: a clinical review. JAMA 311:1052-1060

2. Hanaoka C, Norton EC (2008) Informal and formal care for elderly persons: how adult children's characteristics affect the use of formal care in Japan. Soc Sci Med 67(6):1002-1008

3. Triantafillou J, Naiditch M, Repkova K et al (2015) Informal care in the long-term care system. European Overview Paper.
European Centre for Social Welfare Policy and Research 2010. http://www.euro.centre.org. Accessed Feb 2015

4. Australian Institute of Health and Welfare (2015) Informal care. http://www.aihw.gov.au/informal-care-ageing. Accessed Feb 2015

5. Schulz R, Beach SR (1999) Caregiving as a risk factor for mortality: the Caregiver Health Effects Study. JAMA 282:2215-2219

6. Gouin JP, Hantsoo L, Kiecolt-Glaser JK (2008) Immune dysregulation and chronic stress among older adults: a review. Neuroimmunomodulation 15:251-259

7. Lee S, Colditz GA, Berkman LF, Kawachi I (2003) Caregiving and risk of coronary heart disease in U.S. women: a prospective study. Am J Prev Med 24:113-119

8. Bushby K, Finkel R, Birnkrant DJ et al (2010) Diagnosis and management of Duchenne muscular dystrophy, part 1: diagnosis, and pharmacological and psychosocial management. Lancet Neurol 9:77-93

9. Bushby K, Finkel R, Birnkrant DJ et al (2010) Diagnosis and management of Duchenne muscular dystrophy, part 2: implementation of multidisciplinary care. Lancet Neurol 9:177-189

10. Eagle M, Bourke J, Bullock R et al (2007) Managing Duchenne muscular dystrophy - the additive effect of spinal surgery and home nocturnal ventilation in improving survival. Neuromuscul Disord 17:470-475

11. Landfeldt E, Lindgren P, Bell C et al (2014) The burden of Duchenne muscular dystrophy: an international, cross-sectional study. Neurology 83:529-536

12. Baiardini I, Minetti C, Bonifacino S et al (2011) Quality of life in Duchenne muscular dystrophy: the subjective impact on children and parents. J Child Neurol 26:707-713

13. Boyer F, Drame M, Morrone I, Novella JL (2006) Factors relating to carer burden for families of persons with muscular dystrophy. J Rehabil Med 38:309-315

14. Chen JY, Chen SS, Jong YJ et al (2002) A comparison of the stress and coping strategies between the parents of children with Duchenne muscular dystrophy and children with a fever. J Pediatr Nurs 17:369-379

15. Abi Daoud MS, Dooley JM, Gordon KE (2004) Depression in parents of children with Duchenne muscular dystrophy. Pediatr Neurol 31:16-19

16. Kenneson A, Bobo JK (2010) The effect of caregiving on women in families with Duchenne/Becker muscular dystrophy. Health Soc Care Community 18:520-528

17. Nereo NE, Fee RJ, Hinton VJ (2003) Parental stress in mothers of boys with Duchenne muscular dystrophy. J Pediatr Psychol 28:473-484

18. Magliano L, Patalano M, Sagliocchi A et al (2014) "I have got something positive out of this situation": psychological benefits of caregiving in relatives of young people with muscular dystrophy. J Neurol 261:188-195

19. Mah JK, Thannhauser JE, Kolski H et al (2008) Parental stress and quality of life in children with neuromuscular disease. Pediatr Neurol 39:102-107

20. Pangalila RF, van den Bos GA, Stam HJ et al (2012) Subjective caregiver burden of parents of adults with Duchenne muscular dystrophy. Disabil Rehabil 34:988-996

21. Samson A, Tomiak E, Dimillo J et al (2009) The lived experience of hope among parents of a child with Duchenne muscular dystrophy: perceiving the human being beyond the illness. Chronic Illn 5:103-114

22. Thomas PT, Rajaram P, Nalini A (2014) Psychosocial challenges in family caregiving with children suffering from Duchenne muscular dystrophy. Health Soc Work 39:144-152

23. Landfeldt E, Lindgren P, Bell C et al (2015) Compliance to care guidelines for Duchenne muscular dystrophy. J Neuromuscul Dis $2: 63-72$ 
24. Bladen CL, Rafferty K, Straub V et al (2013) The TREAT-NMD Duchenne muscular dystrophy registries: conception, design and utilisation by industry and academia. Hum Mutat 34:1449-1457

25. Dolan P (1997) Modeling valuations for EuroQol health states. Med Care 35:1095-1108

26. Ware JE, Kosinski M, Turner-Bowker DM, Gandek B (2002) How to score version 2 of the SF-12 health survey. Lincoln, RI: Quality Metric Incorporated

27. Zarit SH, Reever KE, Bach-Peterson J (1980) Relatives of the impaired elderly: correlates of feelings of burden. Gerontologist 20:649-655

28. Kind P, Hardman G, Macran S (1999) UK Population Norms for EQ-5D. Discussion paper, p 172. Centre for Health Economics, The University of York

29. Kind P, Dolan P, Gudex C, Williams A (1998) Variations in population health status: results from a United Kingdom national questionnaire survey. BMJ 316:736-741

30. Perrin JM, Bloom SR, Gortmaker SL (2007) The increase of childhood chronic conditions in the United States. JAMA 297:2755-2759

31. Wise PH (2007) The future pediatrician: the challenge of chronic illness. J Pediatr 151:S6-S10

32. Whittemore R, Jaser S, Chao A et al (2012) Psychological experience of parents of children with type 1 diabetes: a systematic mixed-studies review. Diabetes Educ 38:562-579

33. Khanna R, Jariwala K, Bentley JP (2013) Health utility assessment using EQ-5D among caregivers of children with autism. Value Health 16:778-788
34. Cousino MK, Hazen RA (2013) Parenting stress among caregivers of children with chronic illness: a systematic review. J Pediatr Psychol 38:809-828

35. Sullivan PW, Slejko JF, Sculpher MJ, Ghushchyan V (2011) Catalogue of EQ-5D scores for the United Kingdom. Med Decis Making 31:800-804

36. Walters SJ, Brazier JE (2005) Comparison of the minimally important difference for two health state utility measures: EQ-5D and SF-6D. Qual Life Res 14:1523-1532

37. Kolappa K, Henderson DC, Kishore SP (2013) No physical health without mental health: lessons unlearned? Bull World Health Organ 91:3-3A

38. Wong RK, Drossman DA, Weinland SR, Morris CB, Leserman J, Hu Y, Kelapure R, Bangdiwala SI (2013) Partner burden in irritable bowel syndrome. Clin Gastroenterol Hepatol 11:151-155

39. Naglie G, Hogan DB, Krahn M et al (2011) Predictors of family caregiver ratings of patient quality of life in Alzheimer disease: cross-sectional results from the Canadian Alzheimer's Disease Quality of Life Study. Am J Geriatr Psychiatry 19:891-901

40. Torres AR, Hoff NT, Padovani CR, Ramos-Cerqueira AT (2012) Dimensional analysis of burden in family caregivers of patients with obsessive-compulsive disorder. Psychiatry Clin Neurosci 66:432-441

41. Leroi I, Harbishettar V, Andrews M, McDonald K, Byrne EJ, Burns A (2012) Carer burden in apathy and impulse control disorders in Parkinson's disease. Int J Geriatr Psychiatry 27:160-166 